Research Paper

\title{
MiR27a Promotes the Development of Macrophage-like Characteristics in 3T3-L1 Preadipocytes
}

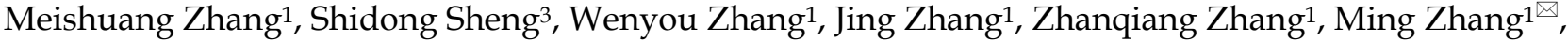 \\ Grant M. Hatch ${ }^{4}$ and Li Chen ${ }^{1,2}$
}

1. Department of Pharmacology, College of Basic Medical Sciences, Jilin University, Changchun 130021, China

2. School of nursing, Jilin University, Changchun 130021, China

3. Department of Surgery, Hepatology Hospital of Jilin Province, Changchun, Jilin, 130021, China

4. Department of Pharmacology \& Therapeutics, Center for Research and Treatment of Atherosclerosis, University of Manitoba, DREAM, Children's Hospital Research Institute of Manitoba, Winnipeg, Manitoba, Canada, R3E 3P4

$\triangle$ Corresponding authors: Dr. Li Chen, Professor and Director, Department of Pharmacology, College of Basic Medical Sciences, School of nursing, Jilin University, 126 Xin Min Street, Changchun, Jilin 130021, China. E-mail: chensubmit@163.com, Tel: +86-0431-85619799 and Dr. Ming Zhang, Associated Professor, Department of Pharmacology, College of Basic Medical Sciences, Jilin University, 126 Xin Min Street, Changchun, Jilin 130021, China. E-mail: zhangming_00@126.com, Tel: +86-0431-85619799

(C) Ivyspring International Publisher. This is an open access article distributed under the terms of the Creative Commons Attribution (CC BY-NC) license (https://creativecommons.org/licenses/by-nc/4.0/). See http://ivyspring.com/terms for full terms and conditions.

Received: 2018.03.25; Accepted: 2018.07.28; Published: 2018.09.07

\begin{abstract}
Recruitment and polarization of classically activated (MI) macrophages within adipose tissue contribute to chronic low-grade inflammation in obesity. Adipose tissue precursor cells exhibit the capacity to develop macrophage-like characteristics and adipocyte-derived miR27a is known to promote reprogramming of somatic cells. It was unknown whether exogenous addition of miR27a promote the development of macrophage-like characteristics of adipose precursor cells. We examined macrophage surface antigen, phagocytosis and migration ability in 3T3-L1 preadipocytes transfected with miR27a mimics. Transfection of 3T3-L1 preadipocytes with miR27a mimics increased phagocytosis and migration and increased the number of cells expressing the macrophage makers F4/80 and MHC compared to controls. M2 and CD206 macrophage markers were unaltered. In addition, transfection of 3T3-L1 preadipocytes with miR27a mimics reduced PPARY expression, activated NF-KB and promoted secretion of the inflammatory cytokines MCP-1, TNF- $\alpha$ and IL-1 $\beta$ compared to controls. The level of anti-inflammatory factors Arg-1, IL-10, Yml and Fizzl were unaltered. Secretion of miR27a was increased in conditioned medium prepared from palmitic acid-treated differentiated 3T3-Ll adipocytes compared to controls. Incubation of 3T3-Ll preadipocytes with this conditioned medium increased phagocytosis and migration compared to controls. Finally, conditioned medium prepared from differentiated 3T3-L1 adipocytes transfection with miR27a inhibitors reduced phagocytosis and migration in 3T3-L1 preadipocytes compared to controls. The data indicate that PPARY agonists may reverse the activation of NF-KB pathway mediated by miR27a overexpression and reduce phagocytosis and migration of adipose precursor cells. In addition, miR27a may promote the development of macrophage-like characteristics in 3T3-L1 preadipocytes.
\end{abstract}

Key words: obesity, miR27a, inflammation, preadipocytes, 3 T3 cells, macrophage-like

\section{Introduction}

Adipose tissue serves not only as an energy storage and endocrine organ but additionally as a source of regional immune cells. During the development of obesity macrophages accumulate within adipose tissue. This immune steady-state disorder is considered an initial key factor in the development of obesity-induced insulin resistance ${ }^{[1]}$.

Adipose tissue macrophages are derived both locally and through chemotactic migration ${ }^{[2,3]}$. Local adipocyte progenitor cells may be reprogrammed into macrophage-like cells or present macrophage-like characteristics[4]. In addition, preadipocytes have the capacity to develop phagocytic and antimicrobial abilities subsequent to cell-to-cell contact with peritoneal macrophages or with a broad spectrum of functional Toll-like receptors ${ }^{[5-11]}$. Previously we demonstrated that adipose tissue from obese individuals secreted miR27a and that adipocyte cell 
derived miR27a induced insulin resistance in skeletal muscle ${ }^{[12]}$. In addition, adipose cell derived miR27a induced activation of macrophages in insulin resistant high fat diet fed obese mice through inhibition of PPAR ${ }^{[13]}$. The above studies indicate that during the process of conversion to mature adipocytes, adipose precursor cells may produce cells with functional macrophage-like characteristics. However, it was unknown whether secreted factors from adipose tissue may be involved in adipose precursor cell differentiation into macrophage-like cells.

In the present study, we examined phagocytosis, migration ability and expression of the macrophage markers F4/80, MHC and CD206 in 3T3-L1 preadipocytes transfected with miR27a mimics. In addition, expression of PPAR $\gamma$, activation of NF-KB and secretion of proinflammatory and anti-inflammatory cytokines were examined in these cells. Expression of NF- $\mathrm{kB}$ and phagocytosis and migration ability of adipose precursor cells were examined after PPARY activation. Finally, we examined phagocytosis and migration ability of 3T3-L1 preadipocytes cultured with conditional medium from palmitic acid (PA)-treated differentiated adipocytes. Our results suggested that miR27a promotes the development of macrophagelike characteristics in 3T3-L1 preadipocytes.

\section{Materials and Methods}

\section{Cell culture}

3T3-L1 preadipocytes (ATTC, USA) were cultured in Dulbecco's modified Eagle's medium (DMEM) and $10 \%$ bovine calf serum (Gibco, USA) in an atmosphere of $5 \% \mathrm{CO}_{2}$ at $37^{\circ} \mathrm{C}$. For differentiation studies, 3T3-L1 cells were incubated with DMEM containing 10\% FBS, $1 \mu \mathrm{M}$ dexamethasone (Sigma, USA), $0.5 \mathrm{mM}$ IBMX (Sigma, USA) and $10 \mu \mathrm{g} / \mathrm{ml}$ insulin or in DMEM containing 10\% FBS and 10 $\mu \mathrm{g} / \mathrm{ml}$ insulin or DMEM containing 10\% FBS. Cell culture medium was changed every two days until the 8-10th day.

\section{Transfection of 3T3-L1 preadipocytes with MiR27a mimics}

MiR27a mimics were transfected into 3T3-L1 preadipocytes cells using Lipofectamine 2000 (Invitrogen, USA) as per the manufacturer's instructions. MiR27a mimics (GenePharma, USA) were diluted in DEPC-treated water to a final concentration of $20 \mu \mathrm{M}$ then mixed with transfection reagent in medium, 1:1 ratio, the final transfection concentration of miR27a was $50 \mathrm{pmol} / \mathrm{ml}$. The suspension was incubated for $20 \mathrm{~min}$ at room temperature. The transfection cocktail was then added in a dropwise manner to cells that had reached $60-70 \%$ confluence. Cells were then incubated in 5\% FBS optimal DMEM with transfection mixture for 24 h, 48 h, $72 \mathrm{~h}$. In other studies cells were treated with 20 $\mu \mathrm{M}$ rosiglitazone for a further $48 \mathrm{~h}$ after miR27a was overexpressed. In some experiments, miR27a inhibitors (GenePharma, USA) were diluted in DEPC-treated water to a final concentration of $20 \mu \mathrm{M}$ and cells were then transfected as described above.

\section{Incubation of 3T3-LI preadipocytes with conditional medium}

Culture medium obtained from differentiated 3T3-L1 cells incubated for $48 \mathrm{~h}$ with no additions was defined as the control medium (con). Culture medium obtained from differentiated 3T3-L1 cells incubated for $48 \mathrm{~h}$ with no additions but then subsequently supplemented with exogenous $0.3 \mathrm{mM}$ PA was defined as the conditional medium 1 (CM1). Culture medium obtained from differentiated 3T3-L1 cells incubated with $0.3 \mathrm{mM}$ phosphatidic acid (PA) for 48 $\mathrm{h}$ was defined as the conditional medium 2 (CM2). Culture medium obtained from differentiated 3T3-L1 cells incubated with $0.3 \mathrm{mM}$ PA for $48 \mathrm{~h}$ but then subsequently supplemented with exogenous miR27a inhibitor was defined as conditional medium 3 (CM3). The conditional medium were removed and mixed with an equal volume of DMEM medium (containing $10 \%$ FBS). 3T3-L1 preadipocyte cells were then incubated with the various conditional medium for 48 $\mathrm{h}$ prior to examination of phagocytosis and migration as described below.

\section{Real-time qPCR}

Total RNA from cells was extracted with Trizol reagent (Invitrogen, USA) or from conditioned medium by RNA extraction kit (Qiagen, USA) according to the manufacturer's instructions. After quantification, cDNA was synthesized using a reverse transcription kit (Transgen Biotech, China). Real-time qPCR reaction was performed with cDNA synthesized from $1 \mu \mathrm{g}$ total RNA and miR27a primers (RiboBio, China). The relative abundance of mRNAs was standardized with U6 (RiboBio, China) or miR156a (RiboBio, China) as reference control. PCR primers used for mRNA quantitation are indicated in Table 1 and the expression levels relative to control were determined by calculating $2^{-\triangle \Delta C T}$.

Table 1. Primer sequences

\begin{tabular}{lll}
\hline $\begin{array}{l}\text { Target } \\
\text { gene }\end{array}$ & Forward sense & Reverse sense \\
\hline miR27a & TGCGCTTCACAGTGGCTAAGT & CCAGTGCAGGGTCCGAGGTATT \\
miR156a & GCGGCGGTGACAGAAGAGAGT & CCAGTGCAGGGTCCGAGGTATT \\
U6 & CGCTTCGGCAGCACATATAC & AAATATGGAACGCTTCACGA \\
\hline
\end{tabular}




\section{Neutral red phagocytosis assay}

3T3-L1 preadipocytes cells were suspended in DMEM and adjusted to $1 \times 10^{5}$ cells $/ \mathrm{ml}$ and $500 \mu \mathrm{l}$ of cell suspension seeded in 24-well plates. After $12 \mathrm{~h}$ culture, the cells were transfected with miR27a mimics for 24,48 or $72 \mathrm{~h}$. In other studies cells were treated with $20 \mu \mathrm{M}$ rosiglitazone for a further $48 \mathrm{~h}$ after miR27a was overexpressed. Subsequent to incubation, the supernatant was discarded and $500 \mu \mathrm{l}$ of $0.1 \%$ neutral red solution (Sigma, USA), prepared in physiological saline and filtered, was added to each well. Cells were then incubated at $37^{\circ} \mathrm{C}$ in $5 \% \mathrm{CO}_{2}$ for $1 \mathrm{~h}$ and the neutral red solution removed. Cells were then washed 3 times with pre-warmed PBS. $200 \mu \mathrm{l}$ of cell-lysis solution (0.1 M acetic acid:ethanol, 1:1 v/v) was added to each well. After overnight storage at $4^{\circ} \mathrm{C}$ the absorbance at $540 \mathrm{~nm}$ was measured ( 3 replicates in each experiment group). In some experiments, preadipocytes were incubated for $48 \mathrm{~h}$ with either control (con) or CM1 or CM2 or CM3 medium and phagocytosis determined as above.

\section{Fluorescence microspheres phagocytosis assay}

Fluorescent microspheres (Invitrogen, USA) were activated in 1\% BSA with $80-100 \mathrm{w}$ ultrasound for $5 \mathrm{~min}$, then incubated at $37^{\circ} \mathrm{C}$ for $30 \mathrm{~min}$. 3T3-L1 preadipocytes $\left(2 \times 10^{5}\right.$ cells) were plated to each well of a 6-well plate. After culturing for $12 \mathrm{~h}$, the cells were then transfected with miR27a mimics for $48 \mathrm{~h}$. The supernatant was then removed and cells were incubated at $37^{\circ} \mathrm{C}$ for $1 \mathrm{~h}$ with fluorescent microspheres (cells:fluorescent microspheres, 1:21 ratio). Following incubation, the supernatant was removed and the cells washed 3 times with pre-cooled PBS. Cells were collected, centrifuged at $1,500 \mathrm{rpm}$ for $10 \mathrm{~min}$, resuspended in $350 \mu \mathrm{l}$ of PBS, and then examined by flow cytometry (BD Accuri ${ }^{\circledR}$ C6). Mock transfected 3T3-L1 preadipocytes incubated with fluorescent microspheres as above were used as control. Wells without beads served as the negative control.

\section{Cell surface marker detection}

3T3-L1 preadipocytes cells were transfected with miR27a mimics for $48 \mathrm{~h}$ then washed 3 times with pre-warmed PBS and collected into tubes. The suspension was centrifuged at 1,500 rpm for $10 \mathrm{~min}$ and the cell pellet resuspended in $100 \mu \mathrm{l}$ of PBS. $1 \mu \mathrm{l}$ of F4/80, MHC or CD206 antibody (Biolegend, USA) per $1 \times 10^{6} \mathrm{cells}$ were added and the suspension incubated for $20 \mathrm{~min}$ at $4^{\circ} \mathrm{C}$. After washing once with pre-cooled PBS the suspension was centrifuged at $1,500 \mathrm{rpm}$ for $10 \mathrm{~min}$ and the pellet resuspended in $100 \mu \mathrm{l}$ of PBS. Cells were then examined by flow cytometry (BD Accuri $^{\circledR}$ C6). Mock transfected 3T3-L1 preadipocytes labeled with antibody as above were used as control. In Figure 2 and Figure 3, 3T3-L1 cells incubated in the absence of antibody are shown as the negative control.

\section{Cell migration assay in Transwell ${ }^{\circledR}$ plates}

3T3-L1 preadipocytes cells were transfected with miR27a mimics for $48 \mathrm{~h}$. In other studies cells were treated with $20 \mu \mathrm{M}$ rosiglitazone for a further $48 \mathrm{~h}$ after miR27a was overexpressed. In the conditioned medium (CM) intervention groups, preadipocytes were incubated for $48 \mathrm{~h}$ with either control (con) or CM1 or CM2 or CM3 medium as prepared above. Cells were then seeded in the upper chamber of Transwell ${ }^{\circledR}$ plates (Corning, USA) at a concentration of $1 \times 10^{5}$ cells/dish. The seeding volume was $100 \mu 1$. $600 \mu \mathrm{l}$ of medium containing $10 \%$ fetal bovine serum was added to the lower chamber. The plates were then incubated for up to $10 \mathrm{~h}$. Subsequent to incubation, cells were fixed with absolute methanol and then stained for $20 \mathrm{~min}$ with crystal violet. Cell counting was conducted using a light microscope. The number of cells in five fields were determined.

\section{Inflammatory marker determination}

3T3-L1 preadipocytes cells were transfected plus or minus miR27a mimics for $48 \mathrm{~h}$ and aliquots were collected from the medium and the inflammatory markers MCP-1, TNF- $\alpha$, IL-1 $\beta$ and the anti-inflammatory cytokines Arg-1, IL-10, Ym1 and Fizz1 were measured using ELISA (R\&D, USA) as per the manufacturer's instructions.

\section{Immunoblot analysis}

Proteins were extracted from cell lysates following the manufacturer's protocols (Beyotime, China). Protein concentration was quantified using the BCA protein assay kit (Thermo Fisher Scientific, USA) and $30 \mu \mathrm{g}$ protein was separated in a $12 \%$ SDS polyacrylamide gel and electro transferred onto polyvinylidene difluoride (PVDF) membranes (Bio-Rad, USA). Membranes were blocked with 5\% $(\mathrm{w} / \mathrm{v})$ BSA for $2 \mathrm{~h}$ at room temperature and then incubated with primary antibodies with light shaking overnight at $4^{\circ} \mathrm{C}$. Primary antibodies against PPARY, NF- $\mathrm{kB}, \mathrm{pNF}-\mathrm{kB}, \mathrm{I} \kappa \mathrm{Ba}$ and GAPDH (abcam) were diluted to a ratio of $1: 1000$ in TBST buffer. The membranes were washed 3 times for 5 min each with $10 \mathrm{ml}$ of TBST (10 mM Tris-HCl, $150 \mathrm{mM} \mathrm{NaCl}$ and $0.1 \%(\mathrm{v} / \mathrm{v})$ Tween-20) and then incubated with secondary antibody at room temperature for $2 \mathrm{~h}$. Secondary antibodies goat anti-rabbit or goat anti-mouse (Proteintech, USA) were diluted to a ratio of 1:5000 in TBST buffer. The membrane was incubated in Western ECL substrate (Thermo fisher or 
Proteintech, USA) and exposed to Tanon imager, using Quantity One software for image analyses.

\section{Statistical analysis}

All the data are presented as mean \pm SEM. Student's t-test or one-way AVONA was used for the determination of statistical significance. The level of significance was defined as $\mathrm{p}<0.05$.

\section{Results}

\section{Phagocytic activity is increased in miR27a transfected 3T3-L1 preadipocytes}

The ability to phagocytose particles is a nonspecific immune function of macrophages. To investigate whether miR27a promoted phagocytic activity in 3T3-L1 preadipocyte cells, phagocytosis of neutral red or fluorescent microspheres were determined in cells transfected with or without miR27a mimics. Transfection of cells with miR27a mimics resulted in an approximately 210-fold $(p<0.001)$ increase in cellular miR27a by $24 \mathrm{~h}$ of transfection compared to controls and the levels remained elevated for up to $72 \mathrm{~h}$ (Figure 1A, 1B). Phagocytosis of neutral red was elevated $52 \%$ $(\mathrm{p}<0.001)$ by $48 \mathrm{~h}$ post-transfection with miR27a mimics compared to control (Figure 1C). In addition, phagocytosis of microspheres was increased 2.1-fold $(\mathrm{p}<0.01)$ by $48 \mathrm{~h}$ post-transfection with miR27a mimics compared to control (Figure 1D, 1E). Thus, expression of miR27a mimics in 3T3-L1 preadipocyte cells promotes phagocytosis.

\section{Expression of macrophage antigen $F 4 / 80$, MHC and CD206 in miR27a transfected 3T3-L1 preadipocytes}

To determine if miR27a expression promoted macrophage-like characteristics in 3T3-L1 preadipocyte cells, cells were transfected with or without miR27a mimics and the number of F4/80 (marker of macrophages and macrophage-like cells), MHC (surface-specific antigen of M1 macrophages) and CD206 (surface-specific antigen of M2 macrophages) positive cells determined using flow cytometry. Transfection of 3T3-L1 preadipocyte cells with miR27a mimics resulted in a 38\% $(\mathrm{p}<0.05)$ increase in F4/80 positive cells (Figure 2). MHC and $\mathrm{F} 4 / 80$ double staining indicated that $92 \%(\mathrm{p}<0.05)$ of F4/80 positive cell express MHC compared to control (Figure 3A/B), but the CD206 and F4/80 double staining was unaltered (Figure 3C/D).

\section{Cell migration is enhanced in $\mathrm{miR27a}$ transfected 3T3-LI preadipocytes}

Inflammatory cells including macrophages exhibit the ability to migrate. To determine if miR27a expression promoted migration of 3T3-L1 preadipocytes, cells were transfected with or without miR27a and migration in Transwell ${ }^{\circledR}$ plates determined.There are about $30 \%(p<0.001)$ increase in
A
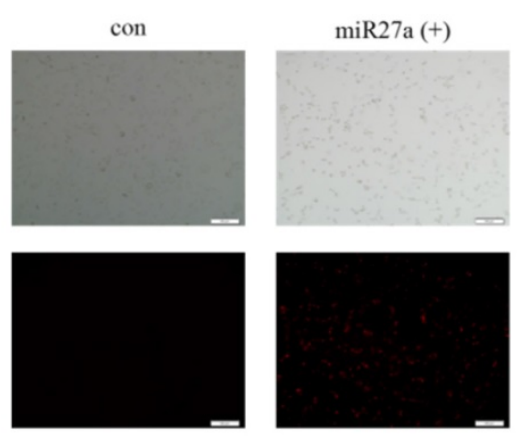

D

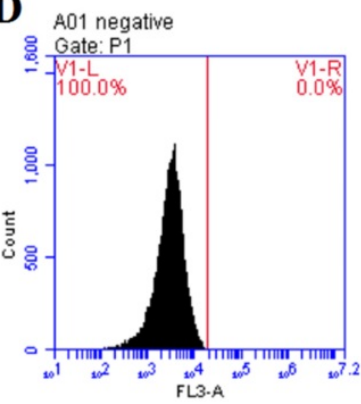

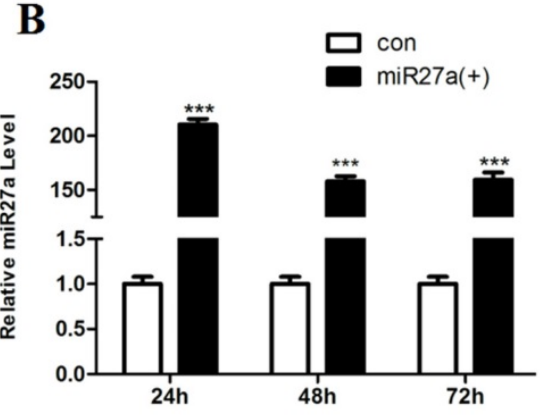

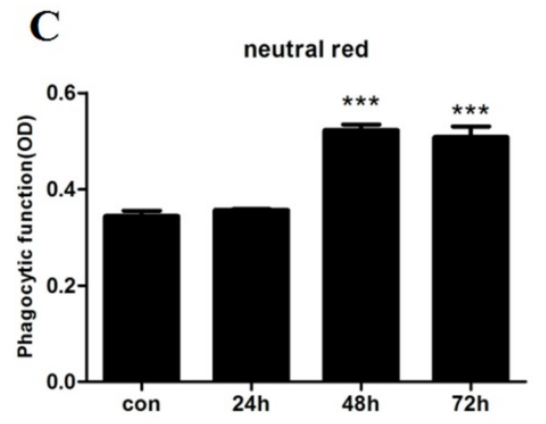

$\mathbf{E}$
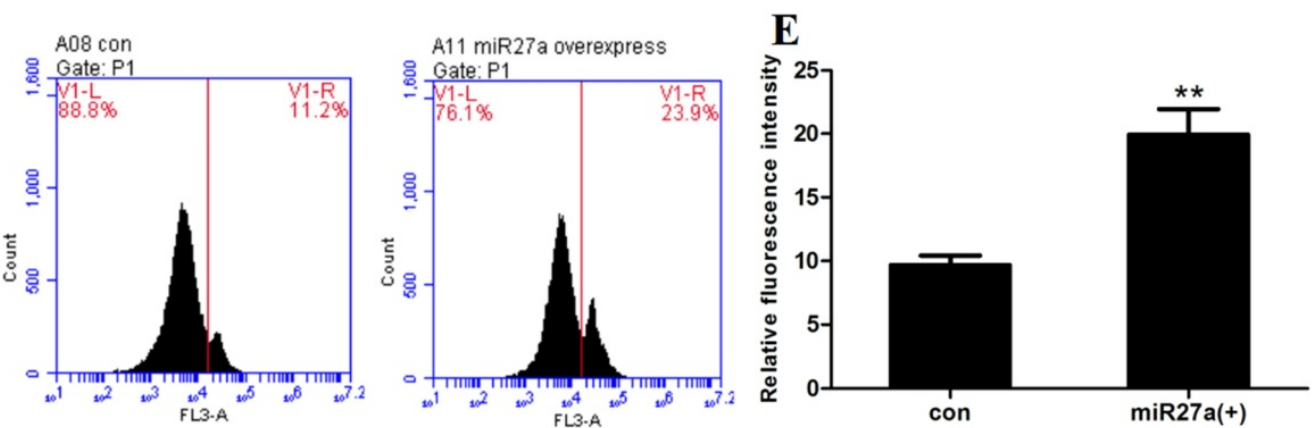

Figure 1. miR27a promotes phagocytosis in 3T3-L1 preadipocytes. A. Transfection efficiency of miR27a in control (con) and miR27a mimic (miR27a(+)) incubated cells. Red fluorescence was the miR-27a transfected in cells. B. Relative expression of miR27a in control (con) and miR27a mimic (miR27a(+)) incubated cells. Data are shown as the mean \pm SEM, $n=6, * * * p<0.001$ compared to control. C. Phagocytic activity in control (con) and cells transfected with miR27a mimic for up to $72 \mathrm{~h}$. $\mathrm{n}=5$, $* * * \mathrm{p}<0.001$ compared to control. D. and E. Phagocytic activity in control (con) and cells transfected with miR27a mimic (miR27a(+)) using flow cytometry. $\mathrm{n}=3$, ** $<<0.01$, compared to control. 

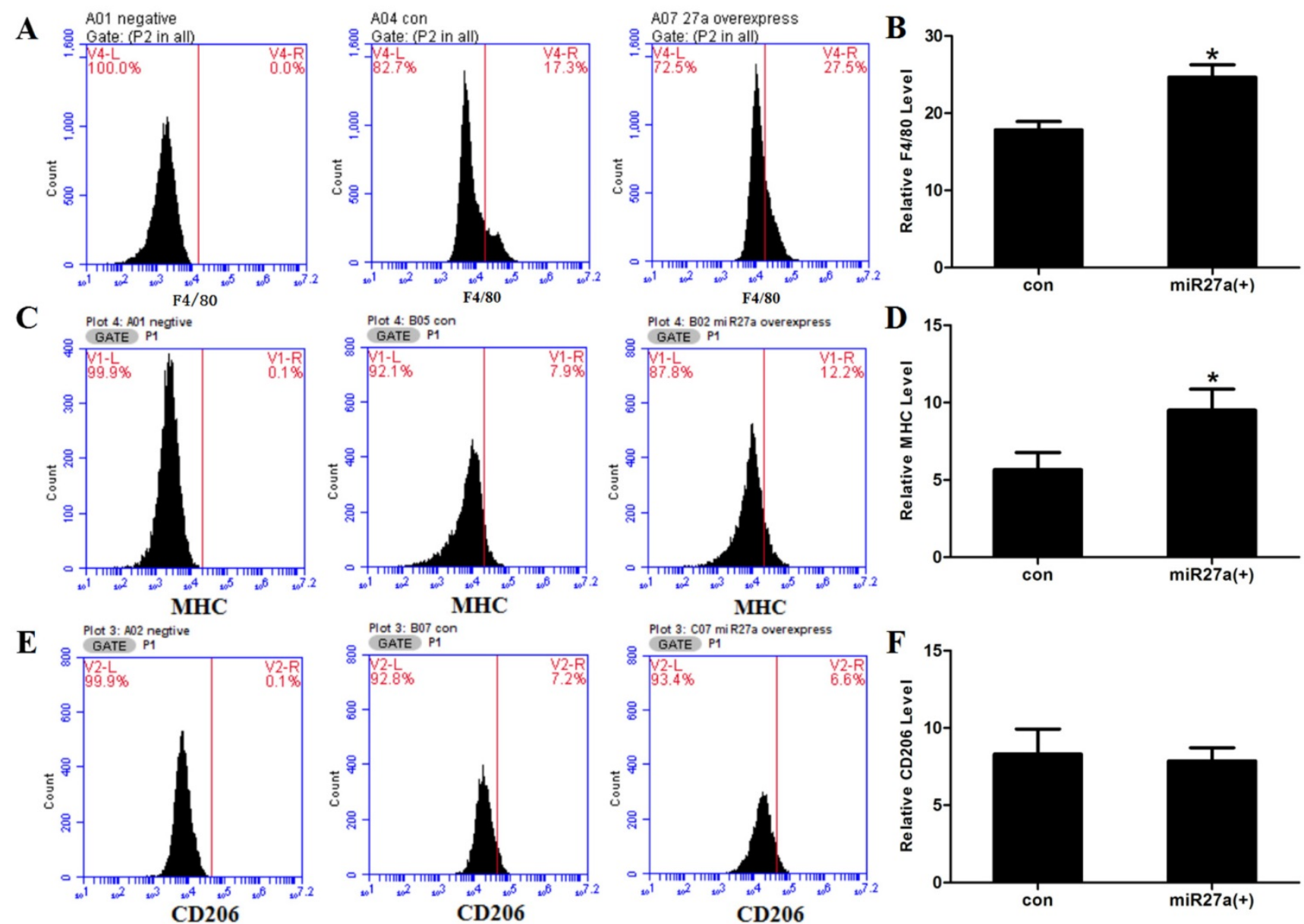

Figure 2. miR27a influences expression of the macrophage cell surface marker. A. Cell surface F4/80 expression in control (A04 con) and miR27a mimic (A07 27a overexpress) incubated cells. Negative control (A01 negative). B. Relative F4/80 level in control (con) and miR27a mimic (miR27a(+)) incubated cells. C. Cell surface MHC expression in control (B05 con) and miR27a mimic (B02 27a overexpress) incubated cells. Negative control (A01 negative). D. Relative MHC level in control (con) and miR27a mimic $(\mathrm{miR} 27 \mathrm{a}(+))$ incubated cells. E. Cell surface CD206 expression in control (B07 con) and miR27a mimic (C07 27a overexpress) incubated cells. Negative control (A02 negative). F. Relative CD206 level in control (con) and miR27a mimic (miR27a(+)) incubated cells.

migration of 3T3-L1 preadipocytes was observed as early as $6 \mathrm{~h}$ in miR27a mimics transfected cells compared to control (Figure 4A, 4B). Thus, miR27a transfection of 3T3-L1 preadipocytes enhances cell migration.

\section{Inflammatory signaling and cytokine secretion is increased in miR27a transfected 3T3-LI preadipocytes}

To determine if miR27a expression in 3T3-L1 preadipocytes modulates secretion of proinflammatory and anti-inflammatory cytokines, the level of MCP-1, TNF- $\alpha$, IL-1 $\beta$, Arg-1, IL-10, Ym1 and Fizz1 were determined in the culture medium of cells transfected with or without miR27a mimics. The level of MCP-1, TNF- $\alpha$ and IL-1 $\beta$ were increased $17 \%$ $(p<0.05), 13 \%(p<0.05)$ and $10 \%(p<0.01)$, respectively, in the supernatant of 3T3-L1 preadipocytes transfected with miR27a mimics compared to control (Figure 5A/B/C). In contrast, expression of the M2 polarization cytokine Arg-1, IL-10, Ym1 and Fizz1 were unaltered in the supernatant of 3T3-L1 preadipocytes transfected with miR27a mimics compared to control (Figure 5D/E/F/G). Thus, miR27a expression in 3T3-L1 preadipocytes increases secretion of inflammatory cytokines into the medium.

The expression of TNF- $\alpha$, IL- $1 \beta$ and MCP- 1 are regulated by the proinflammatory transcription factor, NF-kB. Activation of the nuclear receptor PPARY is known to suppress NF-KB activity and activation and IкBa degradation results in NF- $\mathrm{KB}$ inactivation. To determine if miR27a expression in 3T3-L1 preadipocytes modulates inflammatory signaling the level of PPAR $\gamma, \mathrm{NF}-\mathrm{\kappa B}, \mathrm{pNF}-\mathrm{kB}$, and IкBa were determined in cells transfected with or without miR27a mimics. PPARY expression in miR27a transfected 3T3-L1 preadipocytes was decreased $66 \%$ $(\mathrm{p}<0.01)$ compared to control (Figure 5H, 5I). In addition, expression of IkBa was decreased $34 \%$ $(\mathrm{p}<0.05)$ in miR27a transfected cells compared to control. In contrast, expression of $\mathrm{p}-\mathrm{NF}-\mathrm{kB}$ was increased 3.1-fold $(\mathrm{p}<0.05)$ in miR27a transfected 3T3-L1 preadipocytes compared to control. The total level of NF-kB was unaltered. Thus, miR27a 
expression in 3T3-L1 preadipocytes promotes proinflammatory signaling.

\section{PPARY activation inhibits phagocytic and migration activity and inflammatory pathway protein expression in miR27a transfected 3T3-LI preadipocytes}

To further confirm the roles of PPARY and NF-kB in miR27a regulated phagocytosis and migration ability, 3T3-L1 preadipocytes cells were treated with or without rosiglitazone $(20 \mu \mathrm{M})$ for $48 \mathrm{~h}$ after miR27a mimics transfection. At $6 \mathrm{~h}$, a $38 \%$ $(p<0.001)$ decrease in migration of 3T3-L1 preadipocytes was observed in rosiglitazone treated cells compared to miR27a transfected cells. (Figure $6 \mathrm{~A}, 6 \mathrm{~B})$. After $48 \mathrm{~h}$, phagocytosis of neutral red was reduced $17 \%(p<0.001)$ in miR27a transfected cells treated with rosiglitazone compared to miR27a transfected cells (Figure 6C). PPARY and IKBa expression were increased 1.53-fold $(\mathrm{p}<0.001)$ and
A

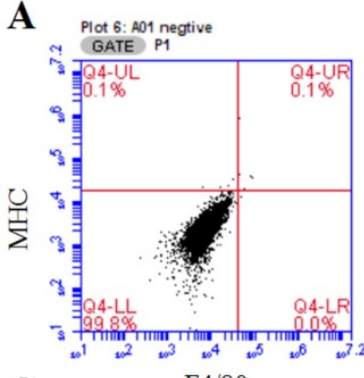

C

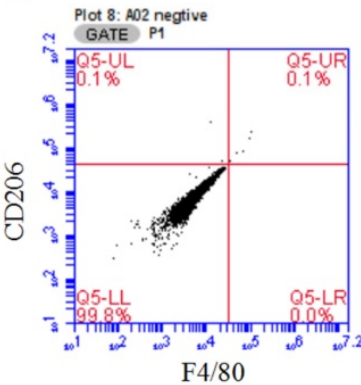

Plot 6: B07 con
GATE P1

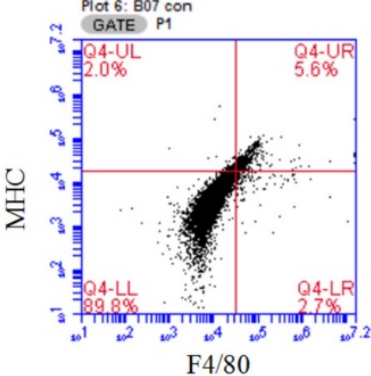

F $4 / 80$

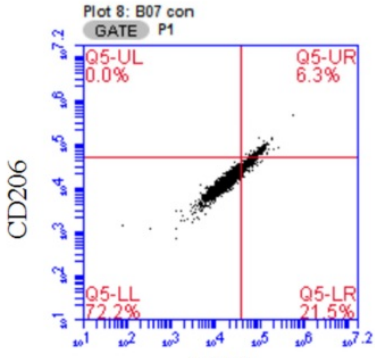

$\mathrm{F} 4 / 80$

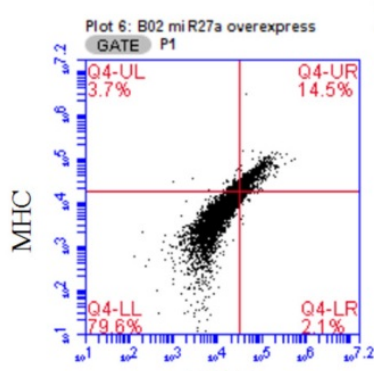

$\mathrm{F} 4 / 80$

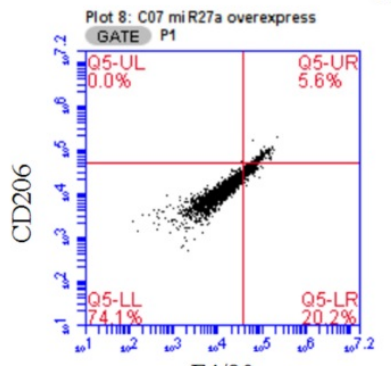

$\mathrm{F} 4 / 80$
B

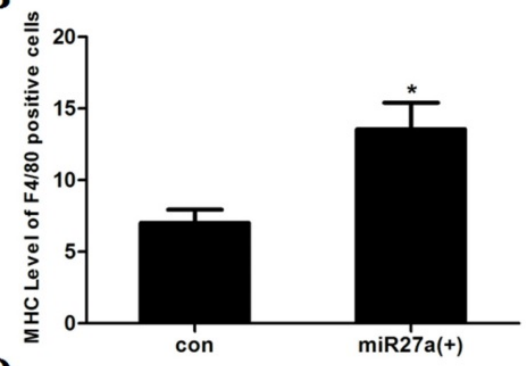

D

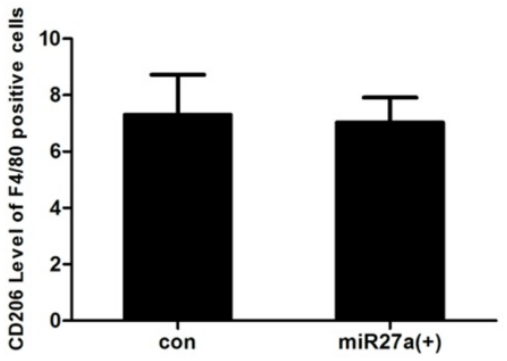

Figure 3. Transfection of 3T3-L1 preadipocyte cells with miR27a mimics increases the number of F4/80 positive cells expressing MHC. A. Cell surface MHC expression in control (B07 con) and miR27a mimic (B02 miR27a overexpressing) incubated cells. Negative control (A01 negative). B. Relative MHC level of F4/80 positive cells in control (con) and miR27a mimic (miR27a(+)) incubated cells. C. Cell surface CD206 expression in control (B07 con) and miR27a mimic (C07 miR27a overexpress) incubated cells. Negative control (A02 negative). D. Relative CD206 level of F4/80 positive cells in control (con) and miR27a mimic (miR27a $(+))$ incubated cells. $n=3,{ }^{*} p<0.05$, compared to control.

A

$6 \mathrm{~h}$
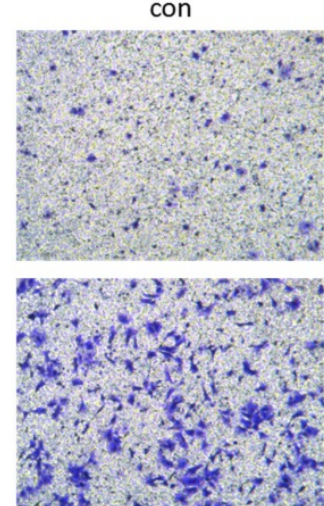

$8 \mathrm{~h}$

$10 \mathrm{~h}$
$\operatorname{miR} 27 \mathrm{a}(+)$
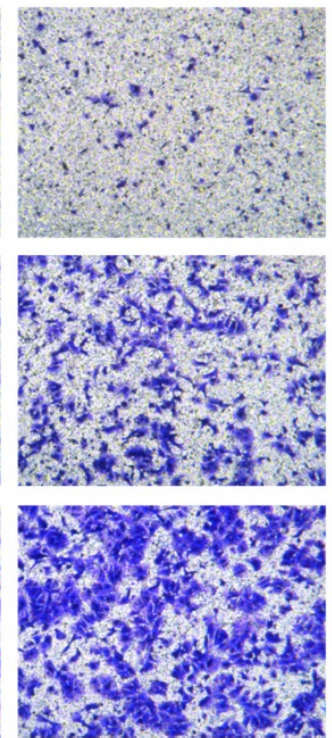

B

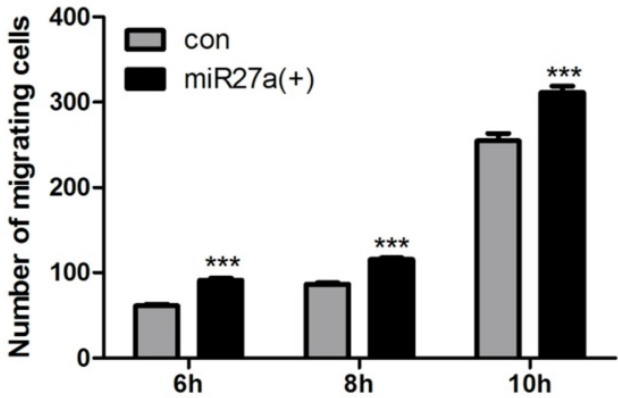

Figure 4. miR27a enhances 3T3-L1 preadipocytes cell migration. A. Cells were cultured on Transwell ${ }^{\circledR}$ plates and incubated in the absence (con) or presence of miR27a mimic $(\mathrm{miR} 27 \mathrm{a}(+))$ for up to $10 \mathrm{~h}$ and stained with crystal violet. $(100 \times$ magnification $)$. A relative micrograph is depicted. B: The number of migrating cells. $\mathrm{n}=3$, $* * * \mathrm{p}<0.001$, compared to control. 
A

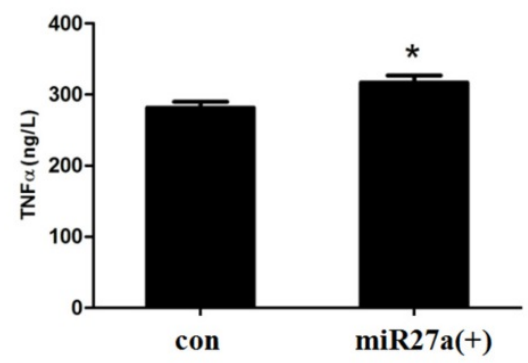

D
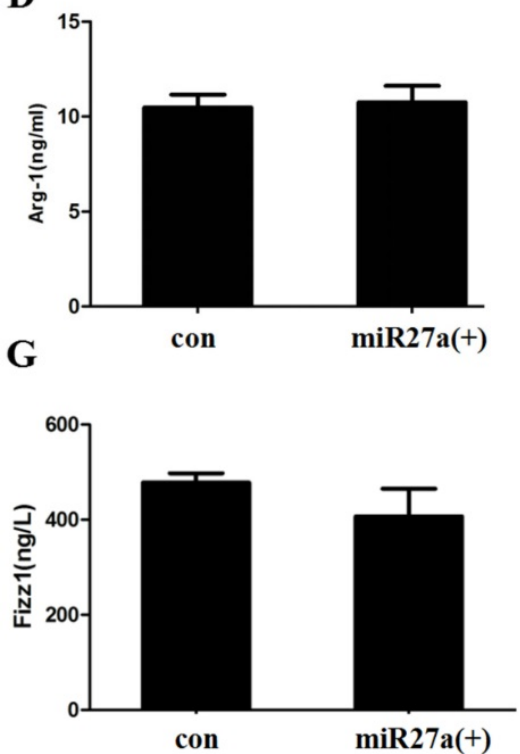

B

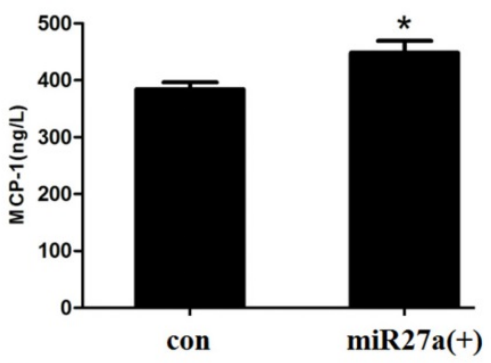

E
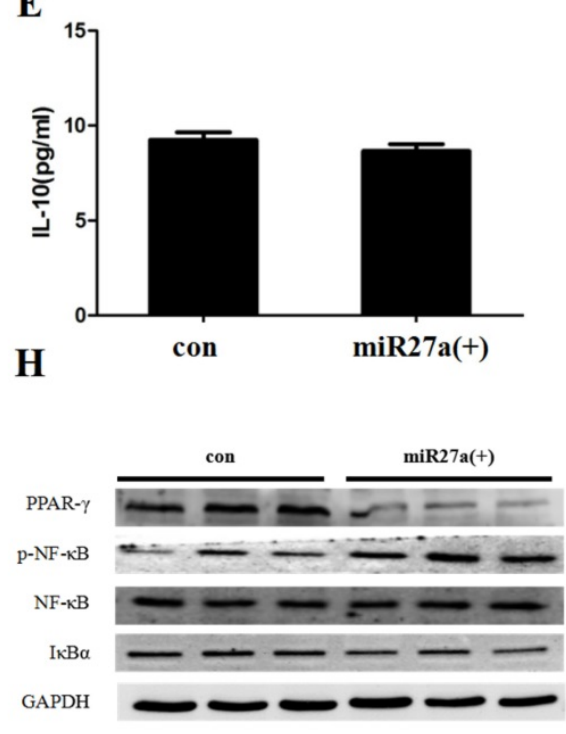

C

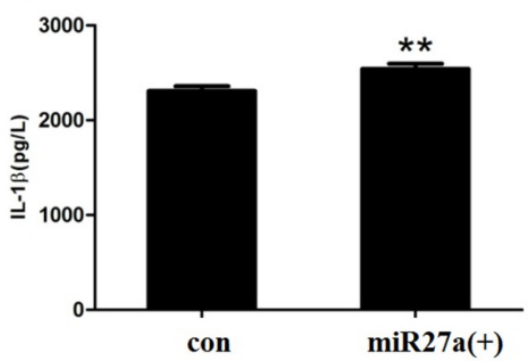

F

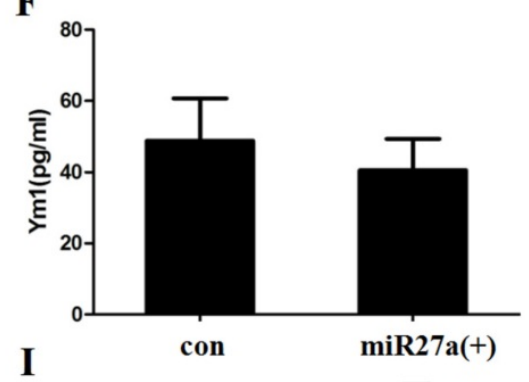

Figure 5. miR27a enhances secretion of inflammatory factors and modulates inflammatory signaling in 3T3-L1 preadipocytes. The level of TNFa (A), MCP-1 (B), IL-1 (C), Arg-1 (D), IL-10 (E), Yml (F) and Fizzl (G) in control (con) and miR27a mimic (miR27a(+)) incubated cells. $n=6$, * $p<0.05$, ${ }^{* *} \mathrm{p}<0.01$, compared to control. $\mathrm{H}$. Western blot analysis of PPARY, NF-KB, P-NF-KB and IKBa in control (con) and miR27a mimic (miR27a(+)) incubated cells. A representative blot is depicted. I. Relative protein expression of PPARY, NF-KB, $\mathrm{p}-\mathrm{NF}-\mathrm{KB}$ and IKBa in control (con) and miR27a mimic (miR27a(+)) incubated cells. $\mathrm{n}=3,{ }^{*} \mathrm{p}<0.05,{ }^{* *} \mathrm{p}<0.01$, compared to control.

$47 \%(\mathrm{p}<0.01)$, respectively, in rosiglitazone treated miR27a mimic transfected 3T3-L1 preadipocytes compared to only miR27a transfected 3T3-L1 preadipocytes. In contrast, expression of $\mathrm{p}-\mathrm{NF}-\mathrm{\kappa B}$ was decreased 5.0-fold $(\mathrm{p}<0.001)$ in rosiglitazone treated 3T3-L1 preadipocytes compared to miR27a transfected 3T3-L1 preadipocytes (Figure 6D, 6E). These results indicate that PPARY is a downstream target of miR27a and its activation inhibits the development of macrophage-like characteristics in 3T3-L1 preadipocytes via the NF-kB pathway.

\section{MiR27a derived from PA-treated adipocytes promotes phagocytic activity and migration of 3T3-LI preadipocytes}

We previously demonstrated that addition of PA promotes hypertrophy in adipocytes and that miR27a may be derived from hypertrophic adipocytes in the obese state[12]. To determine the direct effect of adipocyte derived miR27a on the development of macrophage characteristics in 3T3-L1 preadipocyte cells, 3T3-L1 preadipocytes were incubated for $48 \mathrm{~h}$ with conditional medium prepared from differentiated 3T3-L1 adipocytes incubated under various conditions (con, CM1, CM2 and CM3) and phagocytosis and cell migration were subsequently examined. Secretion of miR27a into the medium was increased 2.3-fold $(\mathrm{p}<0.05)$ in culture medium from differentated adipocytes treated with PA (CM2) compared to control (Figure 7A). In contrast, secretion of miR27a into the medium was decreased $98.5 \%$ $(p<0.001)$ in culture medium from differentated adipocytes treated with PA and with miR27a inhibitor (CM3) compared to control. Phagocytosis of neutral red was increased $13 \%(\mathrm{p}<0.001)$ in CM2 incubated cells compared to control (Figure 7B). In contrast, phagocytosis of neutral red was decreased $9.1 \%$ in CM3 incubated cells compared to control. Phagocytosis did not differ between cells incubated with either CM1 or control medium indicating that the presence of PA itself in the medium did not indirectly affect phagocytosis. Cell migration was increased $24.5 \%(\mathrm{p}<0.001)$ in CM2 incubated cells compared to control (Figure 7C, 7D). In contrast, cell migration was decreased $18.9 \%$ in CM3 incubated cells compared to control. Cell migration did not 
A

$6 \mathrm{~h}$

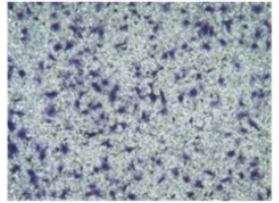

$8 \mathrm{~h}$

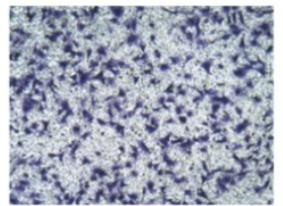

$10 \mathrm{~h}$

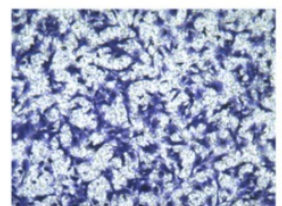
$\operatorname{miR27a}(+)$ :

Rosiglitazone :

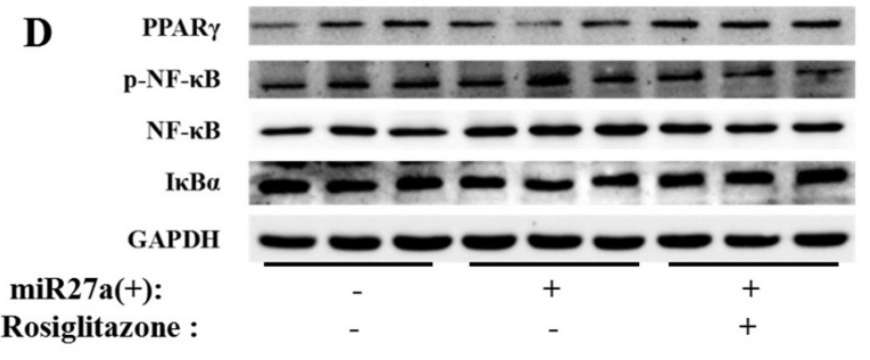

B

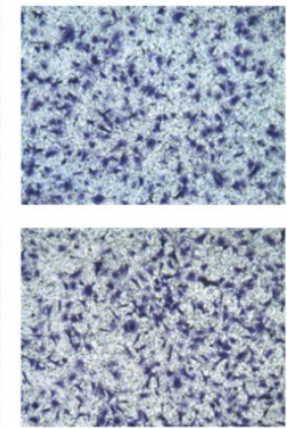

C
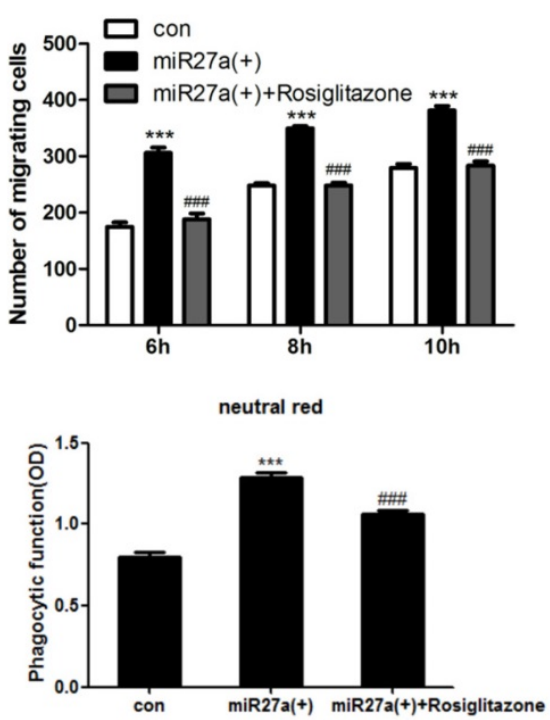

E

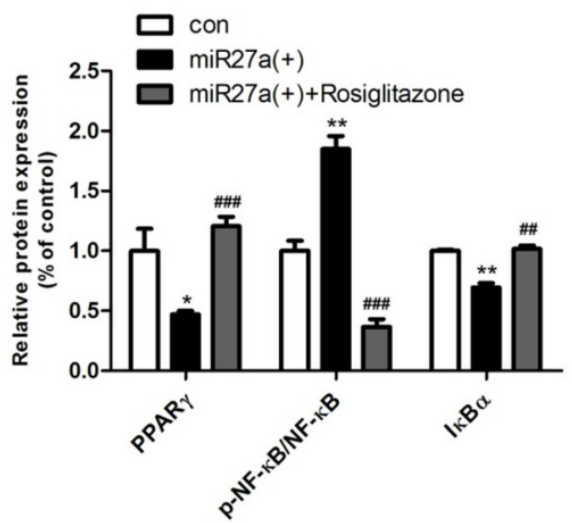

Figure 6. The PPARY agonist rosiglitazone inhibits phagocytic and migration ability and inflammatory pathway protein expression in miR27a transfected 3T3-L1 preadipocytes. A. Cells were cultured on Transwell ${ }^{\circledR}$ plates and incubated in the absence (con) or presence of miR27a mimic (miR27a $(+)$ ) or plus or minus $20 \mu M$ rosiglitazone after miR27a overexpression for up to $10 \mathrm{~h}$ and stained with crystal violet. (100 x magnification). A representative micrograph is depicted. B. The number of migrating cells. Control (con), cells transfected with miR27a mimic or cells transfected with miR27a mimic treated with $20 \mu$ M rosiglitazone $n=3$, *** $p<0.001$, compared to control. \# $\mathrm{p}<0.001$, compared to miR27a transfected cells. $C$. Phagocytic activity in control (con) or cells transfected with miR27a mimic or cells transfected with miR27a mimic plus $20 \mu M$ rosiglitazone. $n=5$, $* * * p<0.001$ compared to control. \#\# $<0.001$, compared to miR27a transfected cells. D. Western blot analysis of PPARy, NF-KB, P-NF-KB and IKBa in control (con) and miR27a mimic treated cells $(\mathrm{miR} 27 \mathrm{a}(+))$ incubated plus or minus $20 \mu \mathrm{M}$ rosiglitazone. A representative blot is depicted. E. Relative protein expression of PPARY, NF-KB, $\mathrm{P}-\mathrm{NF}-\mathrm{KB}$ and IKBa in control (con) and miR27a mimic (miR27a $(+))$ treated cells or miR27a mimic treated cells $(\mathrm{miR} 27 \mathrm{a}(+))$ incubated plus $20 \mu \mathrm{M}$ rosiglitazone. $\mathrm{n}=3$, ${ }^{*} \mathrm{p}<0.05$, ${ }^{* *} \mathrm{p}<0.01$, compared to control.. ${ }^{\mathrm{p}}<0.01, \ldots \ldots \mathrm{p}<0.001$, compared to miR27a transfected cells.

differ between cells incubated with either CM1 or control medium indicating that the presence of PA itself in the medium did not indirectly affect cell migration. Thus, miR27a derived from hypertropic adipocytes promotes phagocytic activity and migration of 3T3-L1 preadipocytes.

\section{Discussion}

Chronic inflammation plays a key role in the development of obesity-induced insulin resistance and macrophage accumulation and infiltration in adipose tissue may contribute significantly to this process $^{[1]}$. It was previously demonstrated that local progenitor cells can be reprogrammed into macrophage-like cells and present macrophage-like characteristics ${ }^{[4]}$. Hence, development of macrophage-like characteristics in adipose precursor cells may promote chronic inflammation in adipose tissue. We previously demonstrated that adipose derived miR27a is a key regulator of macrophage polarization and recruitment in the process of adipose tissue chronic inflammation ${ }^{[13]}$. In the current study, we demonstrated that miR27a plays an important role in the process of adipose precursor cells presentation of macrophage-like characteristics. The principal findings of our study are 1. miR27a promotes macrophage-like characteristics in 3T3-L1 preadipocytes; 2 . miR27a promotes inflammatory signaling and proinflammatory cytokine secretion in 3T3-L1 preadipocytes and, 3. miR27a derived from PA-treated hypertrophic adipocytes may contribute to the promotion of macrophage-like characteristics of preadipocytes. 
A

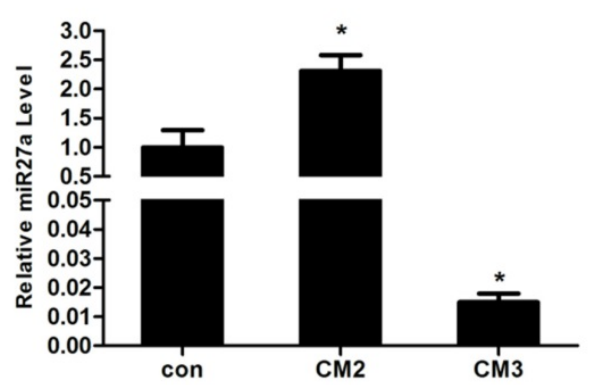

C

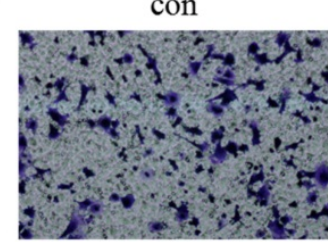

$\mathrm{CM} 2$

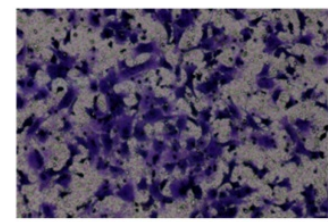

CM1

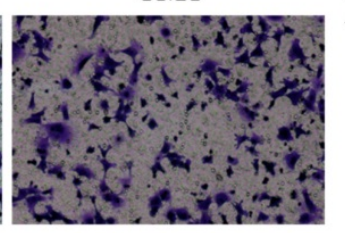

$\mathrm{CM} 3$

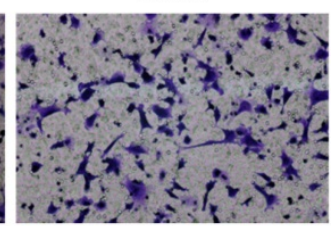

B

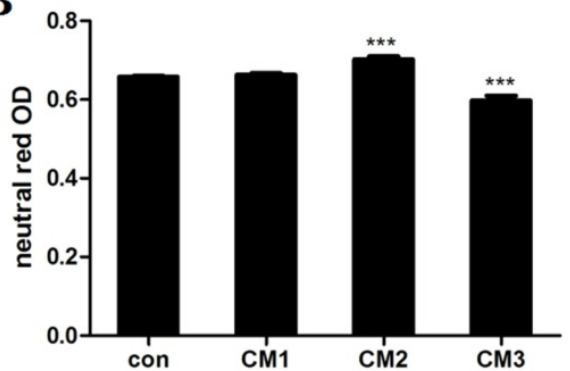

D

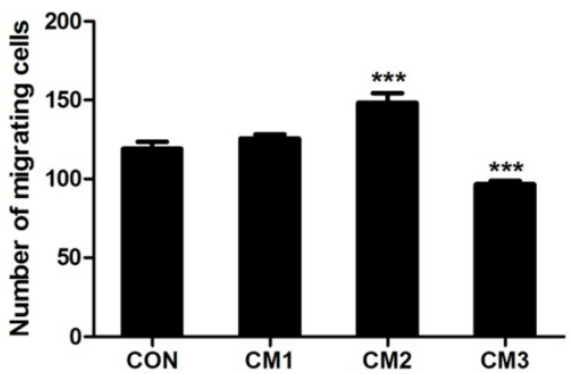

Figure 7. miR27a derived from hypertropic adipocytes promotes phagocytic activity and migration of 3T3-L1 preadipocytes. A. Relative level of miR27a in control (con) or CM2 or CM3 medium. $\mathrm{n}=3$, ${ }^{*} \mathrm{p}<0.05$, compared to control. B. Phagocytic activity in 3T3-L1 preadipocytes incubated with control (con) or CM1 or CM2 or CM3 medium. $\mathrm{n}=3$, *** $\mathrm{p}<0.001$, compared to control. C. Cells cultured on transwell plates and incubated in the presence of control (con) or CM1 or CM2 or CM3 medium and then stained with crystal violet. (100 x magnification). A relative micrograph is depicted. D. The number of migrating cells from $C$. $n=3$, $* * * p<0.001$, compared to control.

A previous study had indicated that preadipocytes may function as macrophage-like cells and raised the possibility of a potential direct involvement of adipose tissue in inflammatory processes ${ }^{[5]}$. In addition, it was shown that preadipocytes exhibit functional features of macrophages, including phagocytosis and anti-microbial activity, suggesting that preadipocyte cells with macrophage activity may play a role in the inflammatory process or immune response ${ }^{[6]}$. However, the regulatory factors involved in this process are unknown. MicroRNAs are a family of non-coding small molecule RNAs containing approximately 20 nucleotides and have the ability to reduce mRNA levels and alter protein expression ${ }^{[14}$, 15]. Previous studies have identified a number of miRNAs are correlated with the preadipocyte phenotype including miR-27, miR- $125 \mathrm{~b}$ or miR-199a ${ }^{[16-18]}$. These miRNAs are involved in regulating preadipocyte differentiation. In addition, miRNAs may modulate somatic macrophage transformation. However, the impact of miRNAs in the development macrophage-like changes in fat precursor cells had not been reported. More interestingly, in the blood of obese individuals miR27a is significantly elevated and miR27a is known to activate type M1 macrophages and promote inflammation [19, 20-22]. In addition, miR27a may play a regulatory role in stem cell and adipocyte differentiation. To our knowledge, no study had investigated whether miR27a could regulate the development of macrophage-like characteristics of adipose precursor cells.

Macrophages exhibit phagocytic capacity and the ability to migrate and secret inflammatory factors involved in the immune response[23-26]. In addition, macrophages classically express multiple surface antigens such as F4/80, MHC and CD206 [27-29]. F4/80 is a common marker of macrophages and macrophage-like cells, MHC is a surface marker of M1 phenotype and CD206 is a surface marker of M2 phenotype. Our results demonstrated that both phagocytic and migratory capacity of adipose tissue precursor cells are significantly enhanced by miR27a expression. Consistent with a previous study ${ }^{[7]}, \mathrm{F} 4 / 80$ shown to be expressed on the plasma membrane of 3T3 preadipocyte cells. In addition, we observed that expression of miR27a increased the number of F4/80 and MHC positive cells. These data clearly indicate that miR27a may promote adipose precursor cells to present macrophage-like characteristics. Our study is the first to demonstrate that miR27a may serve as a mediator for promoting the development of macrophage-like characteristics of 3T3-L1 preadipocyte cells.

Bioinformatic analysis had indicated that downstream targets of miR27a may be associated with the inflammatory response of PPAR family 
members $\left[14,{ }^{30]}\right.$. We previously observed that upregulation of miR27a inhibited transcriptional regulation and protein expression of PPARY in mice[16]. Since PPARY binds to NF-kB directly, it is possible that miR27a might activate NF-KB activity to regulate the expression of inflammatory factors. In the process of adipogenesis, expression of miR27a was shown to be decreased when PPARY was

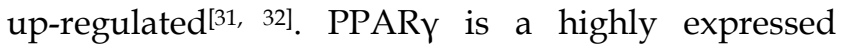
peroxisome proliferator-activated receptor family subtype and directly binds to NF- $\mathrm{kB}$ and inhibits the binding of NF-kB to other target genes. Therefore, inhibition of PPAR $\gamma$ might promote the activation of

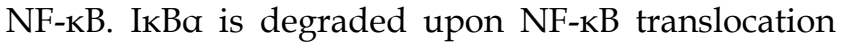
into the nucleus to regulate the expression of inflammatory factors such as TNFa[ ${ }^{33,34]}$. Activation of these signaling proteins promote the secretion of early proinflammatory cytokines such as TNFa, IL-1 $\beta$ and MCP1[35-37]. We observed that expression of miR27a in 3T3 preadipocyte cells activated the inflammatory signaling pathway and increased proinflammatory cytokine secretion. These data further support a role for miR27a in the development of macrophage-like characteristics of preadipocytes. In addition, we observed that PPARY activation with rosiglitazone inhibited the development of macrophage-like characteristics in 3T3-L1 preadipocytes via the NF-kB pathway further indicating that PPARY is a downstream target of miR27a.

Our previous study showed that miR27a is mainly secreted by adipocytes under obese conditions and is capable of regulating insulin resistance in skeletal muscle ${ }^{[12]}$. Whether local adipose tissue secreted miR27a promotes macrophage-like development of adipose precursor cells was unknown. In the current study, we cultured 3T3-L1 preadipocytes with conditional medium derived from differentiated adipocytes incubated with PA with or without miR27a knockdown. The presence of PA in the medium promoted differentiated adipocytes to secrete miR27a. In addition, phagocytosis and migration ability increased significantly after culturing cells in conditioned medium containing miR27a secreted from PA treated differentiated adipocytes. In contrast, knockdown of miR27a in adipocytes reduced phagocytosis and migration ability. Thus, miR27a secreted from mature hypertrophic adipocytes may be a key factor in promoting macrophage-like characteristics of preadipocytes.

In conclusion, miR27a derived from PA-treated hypertrophic adipocytes may promote the development of macrophage-like characteristics in preadipocytes. Since macrophages play a key role in promoting inflammation, miR27a may serve as a potential target for obesity-induced metabolic disorders and local inflammation. Hence, modulation of miR27a expression through an RNA-based treatment may provide a novel therapy for obesity triggered insulin resistance and Type 2 diabetes.

\section{Abbreviations}

miRNA: microRNA; FBS: Fetal Bovine Serum; IBMX: 3-isobutyl-1-methylxanthine; PPARy: peroxisome proliferator-activated receptor gamma; NF-kB: nuclear factor $\mathrm{\kappa B}$; MCP-1: monocyte chemoattractant protein 1; TNFa: tumor necrosis factor alpha; IL-1 $\beta$ : Interleukin-1 Beta; Arg-1: arginase-1; IL-10: Interleukin-10; Ym1: chitinase 3-like 3; Fizz1: Found in inflammatory zone 1; CM: conditional medium; PA: palmitic acid.

\section{Acknowledgments}

This work was supported by funding from the National Natural Science Foundation of China (No. 81570762), the Preclinical Pharmacology R\&D Center of Jilin Province (20170623062TC, 20180201025YY), and Project from The Eduction Department of Jilin Province (JJKH20180247KJ), and Norman Bethune Program of Jilin University (2015224), and the Heart and Stroke Foundation of Canada. GMH is the Canada Research Chair in Molecular Cardiolipin Metabolism.

\section{Author Contributions}

ZMS, CL and ZM have made contributions to conception and design; ZMS, ZJ and ZWY collected data, ZMS, ZWY and ZZQ analysed and interpreted data; ZMS, SSD and GH have been involved in drafting the manuscript and revising it critically for important intellectual content.

\section{Competing Interests}

The authors have declared that no competing interest exists.

\section{References}

[1] Francés DE, Motiño O, Agrá N, et al. Hepatic cyclooxygenase-2 expression protects against diet-induced steatosis, obesity and insulin resistance. Diabetes. 2015; 64: 1522-1531.

[2] Weisberg SP, McCann D, Desai M, et al. Obesity is associated with macrophage accumulation in adipose tissue. J Clin Invest. 2003; 112: 1796-1808.

[3] Curat CA, Miranville A, Sengenès $C$, et al. From Blood Monocytes to Adipose Tissue-Resident Macrophages: Induction of Diapedesis by Human Mature Adipocytes. Diabetes. 2004; 53: 1285-1292.

[4] Katz S, Zsiros V, Dóczi N, et al. GM-CSF and GM-CSF receptor have regulatory role in transforming rat mesenteric mesothelial cells into macrophagelike cells. Inflamm Res. 2016; 65: 827-836.

[5] Cousin B, Munoz O, Andre M, et al. A role for preadipocytes as macrophage-like cells. FASEB J. 1999; 13: 305-312.

[6] Cousin B, André M, Casteilla L, et al. Altered Macrophage-Like Functions of Preadipocytes in Inflammation and Genetic Obesity. J Cell Physiol. 2001; 186: 380-386.

[7] Charrière G, Cousin B, Arnaud E, et al. Preadipocyte Conversion to Macrophage. J Biol Chem. 2003; 278: 9850-9855. 
[8] Wellen KE, Hotamisligil GS. Obesity-induced inflammatory changes in adipose tissue. J Clin Invest. 2003; 112: 1785-1788.

[9] Casteilla L, Dani C. Adipose tissue-derived cells: from physiology to regenerative medicine. Diabetes Metab. 2006; 32: 393-401.

[10] Schäffler A, Schölmerich J, Salzberger B. Adipose tissue as an immunological organ: Toll-like receptors, C1q/TNFs and CTRPs. Trends Immunol. 2007; 28 : 393-399.

[11] Tchkonia T, Morbeck DE, Von Zglinicki T, et al. Fat tissue, aging, and cellular senescence. Aging Cell. 2010; 9: 667-684.

[12] Yu Y, Du H, Wei S, et al. Adipocyte derived exosomal miR-27a induces insulin resistance in skeletal muscle through repression of PPARY. Theranostics. 2018; 8: 2171-2188.

[13] Yao F, Yu Y, Feng L, et al. Adipogenic miR27a in adipose tissue up-regulates macrophage activation via inhibiting PPARY of insulin resistance induced by high-fat diet-associated obesity. Exp Cell Res. 2017; 355: 105-112.

[14] Viesti A Collares R, Salgado W Jr, Pretti da Cunha Tirapelli D, et al. The expression of LEP, LEPR, IGF1 and IL10 in obesity and the relationship with microRNAs. PLoS One. 2014; 9: e93512.

[15] Xie H, Lim B, Lodish HF. MicroRNAs induced during adipogenesis that accelerate fat cell development are downregulated in obesity. Diabetes. 2009; 58: 1050-1057.

[16] Zou B, Ge Z, Zhu W, et al. Persimmon tannin represses 3T3-L1 preadipocyte differentiation via up-regulating expression of miR-27 and down-regulating expression of peroxisome proliferator-activated receptor- $\gamma$ in the early phase of adipogenesis. Eur J Nutr. 2015; 54: 1333-1343.

[17] Ouyang D, Ye Y, Guo D, et al. MicroRNA-125b-5p inhibits proliferation and promotes adipogenic differentiation in 3T3-L1 preadipocytes. Acta Biochim Biophys Sin. 2015; 47: 355-361.

[18] Shi XE, Li YF, Jia L, et al. MicroRNA-199a-5p Affects Porcine Preadipocyte Proliferation and Differentiation. Int J Mol Sci. 2014; 15: 8526-8538.

[19] Butler MG, Wang K, Marshall JD, et al. Coding and noncoding expression patterns associated with rare obesity-related disorders: Prader-Willi and Alstrom syndromes. Adv Genomics Genet. 2015; 2015: 53-75.

[20] Shi C, Huang F, Gu X, et al. Adipogenic miRNA and meta-signature miRNAs involved in human adipocyte differentiation and obesity. Oncotarget. 2016; 7: 40830-40845.

[21] Xie N, Cui H, Banerjee $\mathrm{S}$, et al. MiR27a regulates inflammatory response of macrophages by targeting IL-10. J Immunol. 2014; 193: 327-334.

[22] Min S, Li L, Zhang M, et al. TGF-beta-associated miR27a inhibits dendritic cell-mediated differentiation of Th1 and Th17 cells by TAB3, p38 MAPK, MAP2K4 and MAP2K7. Genes Immun. 2012; 13: 621-631.

[23] Jokinen MP, Morgan DL, Price HC, et al. Immunohistochemical Characterization of Sarcomas in Trp53+/- Haploinsufficient Mice. Toxicol Pathol. 2017; 45: 774-785.

[24] Huang Y, Qi H, Zhang Z, et al. Gut REG3y-Associated Lactobacillus Induces Anti-inflammatory Macrophages to Maintain Adipose Tissue Homeostasis. Front Immunol. 2017; 8: 1063.

[25] Bao Y. The progress of studying the mechanisms of immune cells in the regulation of non-alcoholic fatty liver diseases. Zhonghua Gan Zang Bing Za Zhi. 2017; 25: 553-556.

[26] Biagioli M, Carino A, Cipriani S, et al. The Bile Acid Receptor GPBAR1 Regulates the M1/M2 Phenotype of Intestinal Macrophages and Activation of GPBAR1 Rescues Mice from Murine Colitis. J Immunol. 2017; 199: 718-733.

[27] van Diepen JA, Robben JH, Hooiveld GJ, et al. SUCNR1-mediated chemotaxis of macrophages aggravates obesity-induced inflammation and diabetes. Diabetologia. 2017; 60: 1304-1313.

[28] Zhao T, Li Y, Liu B, et al. Inhibition of histone deacetylase 6 restores innate immune cells in the bone marrow in a lethal septic model. J Trauma Acute Care Surg. 2016; 80: 34-40.

[29] Yamamoto S, Muramatsu M, Azuma E, et al. A subset of cerebrovascular pericytes originates from mature macrophages in the very early phase of vascular development in CNS. Sci Rep. 2017; 7: 3855.

[30] McGregor RA, Choi MS. MicroRNAs in the regulation of adipogenesis and obesity. Curr Mol Med. 2011; 11: 304-316.

[31] Kim SY, Kim AY, Lee HW, et al. MiR27a is a negative regulator of adipocyte differentiation via suppressing PPARgamma expression. Biochem Biophys Res Commun. 2010; 392: 323-328.

[32] Lin $Q$, Gao Z, Alarcon RM, et al. A role of miR-27 in the regulation of adipogenesis. FEBS J. 2009; 276: 2348-2358.

[33] Skaug B, Jiang X, Chen ZJ. The role of ubiquitin in NF-kappaB regulatory pathways. Annu Rev Biochem. 2009; 78: 769-796.

[34] Gloire G, Legrand-Poels S, Piette J. NF-kappaB activation by reactive oxygen species: fifteen years later. Biochem Pharmacol. 2006; 72: 1493-1505.

[35] Mueller CK, Schultze-Mosgau S. Histomorphometric analysis of the phenotypical differentiation of recruited macrophages following subcutaneous implantation of an allogenous acellular dermal matrix. Int J Oral Maxillofac Surg. 2011; 40: 401-407.

[36] Fujiu K, Manabe I, Nagai R. Renal collecting duct epithelial cells regulate inflammation in tubulointerstitial damage in mice. J Clin Invest. 2011; 121: 3425-3441.

[37] Wynn TA, Barron L, Thompson RW, et al. Quantitative assessment of macrophage functions in repair and fibrosis. Curr Protoc Immunol. 2011; Chapter 14:Unit14. 22. 